\title{
Conditionally Suboptimal Filtering in Nonlinear Stochastic Differential System*
}

\author{
Tongjun He, Zhengping Shi \\ College of Mathematics and Computer Science, Fuzhou University, Fuzhou, China \\ E-mail: hetongjun@fzu.edu.cn, shizp@fzu.edu.cn \\ Received January 25, 2011; revised April 27, 2011; accepted April 30, 2011
}

\begin{abstract}
This paper presents a novel conditionally suboptimal filtering algorithm on estimation problems that arise in discrete nonlinear time-varying stochastic difference systems. The suboptimal state estimate is formed by summing of conditionally nonlinear filtering estimates that their weights depend only on time instants, in contrast to conditionally optimal filtering, the proposed conditionally suboptimal filtering allows parallel processing of information and reduce online computational requirements in some nonlinear stochastic difference system. High accuracy and efficiency of the conditionally suboptimal nonlinear filtering are demonstrated on a numerical example.
\end{abstract}

Keywords: Suboptimal Estimate, Conditionally Filter, Nonlinear Stochastic System

\section{Introduction}

Some simple Kalman-Bucy filters [1-4] lead to some conditionally optimal filtering [5-7]. This main idea is that the absolute unconditional optimality is rejected and in a class of admissible estimates with some nonlinear stochastic differential equations or nonlinear stochastic difference equations that can be solved online while receiving the results of the measurements, the optimal estimate is found. In this paper we are interesting in constituting a novel conditionally filtering algorithm addressing estimation problems that suboptimal arise in discrete nonlinear time-varying stochastic difference systems with different types of measurements [8-10]. By a weighted sum of local conditionally nonlinear stochastic filtering estimates, the suboptimal estimate of state of this conditionally nonlinear stochastic filtering is given, thus due to its inherent parallel structure, it can be implemented on a set of parallel processors. The aim of this paper is to give an alternative conditionally suboptimal filtering for that kind of discrete time nonlinear stochastic difference systems. As same as the conditionally optimal filtering, the conditionally suboptimal filtering represents the state estimate as a weighted sum of local conditionally filtering estimates associated with the weights depending only on time instants and being inde-

\footnotetext{
${ }^{*}$ The authors were partially supported by NSFC(11001054) and the
} Found of the Ministry of Education of Fujian Province: JA090022. pendent of current measurements.

\section{Problem Statement for Nonlinear Stochastic Difference Systems}

In [6], consider a nonlinear discrete stochastic system whose state vector $\boldsymbol{X}_{l} \in R^{n}$ is determined by a nonlinear stochastic difference system

$$
\boldsymbol{X}_{l+1}=a_{l} \boldsymbol{X}_{l}+a_{0 l}+\left(c_{0 l}+\sum_{r=1}^{n} c_{r l} \boldsymbol{X}_{l r}\right) V_{l} .
$$

Suppose that the observation vector $Y_{l}$ is composed of $N$ differential types of observation sub-vectors, i.e.,

$$
\boldsymbol{Y}_{l}=\left[\boldsymbol{Y}_{l}^{(i)}, \cdots, \boldsymbol{Y}_{l}^{(N)}\right]^{\mathrm{T}},
$$

where $\left(\boldsymbol{Y}_{l}^{(i)}\right)_{i=1}^{N}$ is determined by the stochastic system,

$$
\boldsymbol{Y}_{l}^{(i)}=b_{l}^{(i)} \boldsymbol{X}_{l}+b_{0 l}^{(i)}+\left(d_{0 l}^{(i)}+\sum_{r=1}^{n} d_{r l}^{i} \boldsymbol{X}_{l r}\right) V_{l},
$$

in (1) and (3), $\boldsymbol{X}_{l r}$ is the $r$ th component of the vector $X_{l}, l$ is discrete time, $a_{l}, c_{0 l}, c_{r l}, b_{l}^{(i)}, d_{0 l}^{(i)}, d_{r l}^{(i)}$ are the matrices, $a_{0 l}, b_{0 l}^{(i)}$ are the constant vectors of the respective dimension, $\left\{V_{l}\right\}$ is a sequence of independent random variables with known distribution. We shall also assume that

1) The state sequence of random variables $\left\{X_{l}\right\}$ and the measurement noise sequence of random variables $\left\{V_{l}\right\}$ are independent of each other, so that 


$$
E\left[V_{i} V_{j}^{T}\right]=0, E\left[X_{i} V_{j}\right]=E X_{i} E V_{j}
$$

2) The random variables $\left\{V_{l}\right\}$ is a sequence of Gaussian white noise with zero mean and covariance matrix

$$
E\left[V_{l} V_{l}^{T}\right]=G_{l}=I \text {. }
$$

Usually, for a fixed $i(1 \leq i \leq N)$, the conditionally optimal filter is to find an estimate $\hat{X}_{l}^{(i)}$ of the random state variable $X_{l}$ at each discrete time $l$ by the measurements of the previous random variables $Y_{1}^{(i)}, \cdots, Y_{l-1}^{(i)}$, here, the class of admissible estimates are defined by the difference equation

$$
\hat{X}_{l}^{(i)}=A^{(i)} \delta_{l-1}^{(i)} \psi_{l-1}^{(i)}\left(U_{l-1}^{(i)}, Y_{l-1}^{(i)}\right)+A^{(i)} \gamma_{l-1}^{(i)}
$$

with a given sequence of the vector structural functions $\psi_{l-1}^{(i)}(u, y)$ and all possible values of the matrices of coefficients $\delta_{l-1}^{(i)}$ and the coefficients vectors $\gamma_{l-1}^{(i)}$. The class of admissible filtering is determined by the given sequence of the structural functions $\psi_{l-1}^{(i)}(u, y)$. Certainly, such an estimate $\hat{X}_{l}^{(i)}$ is required to minimize the mean square error in some sense

$$
E\left\|\hat{X}_{l}^{(i)}-X_{l}\right\|^{2}
$$

at any discrete time $l$. This problem arises from the multi-criteria equation, $\left\{\delta_{l}^{(i)}\right\},\left\{\gamma_{l}^{(i)}\right\}$ can be optimal. Our problem is to find a suboptimal estimate $\hat{X}_{l}^{*}$ at any discrete time $l$ by using $N$ different types of estimates $\hat{X}_{l}^{(1)}, \cdots, \hat{X}_{l}^{(N)}$ such that

$$
E\left\|\hat{X}_{l}^{*}-X_{l}\right\|^{2}
$$

is minimal in some sense.

\section{Construction of Conditionally Suboptimal Nonlinear Filter}

\subsection{Conditionally Nonlinear Filter}

For $i(1 \leq i \leq N)$, we firstly choose a sequence of structural functions

$$
\psi_{l}^{(i)}\left(U_{l}^{(i)}, Y_{l}^{(i)}\right)=\left[\hat{X}_{l}^{(i) T} Y_{l}^{(i) T}\right]^{T},
$$

let $A=I$, and substitute into (5) we can derive that

$$
\hat{X}_{l+1}^{(i)}=\delta_{1 l}^{(i)} \hat{X}_{l}^{(i)}+\delta_{2 l}^{(i)} \hat{Y}_{l}^{(i)}+\gamma_{l}^{(i)},
$$

where $\delta_{l}^{(i)}=\left[\delta_{1 l}^{(i)} \delta_{2 l}^{(i)}\right], \quad \hat{X}_{l}=U_{l}^{(i)}(i=1,2, \cdots, N)$, this is a class of admissible filters. The conditionally optimal nonlinear filtering is consist of (1), (3) and (8), and it is denoted by $I$. Applying conditionally optimal nonlinear filter theory [5] we obtain the following results for this conditionally optimal nonlinear filtering $I$ : If $b_{l}^{(i)} R_{l}^{(i)} b_{l}^{(i) T}+H_{l 22}^{(i)}(i=1, \cdots, N)$ is inverse, then

$$
\begin{gathered}
\delta_{2 l}^{(i)}=\left(a_{l} R_{l}^{(i)} b_{l}^{(i) T}+H_{l 12}^{(i)}\right)\left(b_{l}^{(i)} R_{l}^{(i)} b_{l}^{(i) T}+H_{l 22}^{(i)}\right)^{-1} \\
\delta_{1 l}^{(i)}=a_{l}-\delta_{2 l}^{(i)} b_{l}^{(i)} \\
\gamma_{l}^{(i)}=a_{0 l}-\delta_{1 l}^{(i)} b_{0 l}^{(i)},
\end{gathered}
$$

for any $i(i=1,2, \cdots, N)$, where

$$
\begin{aligned}
H_{l 11}= & c_{0 l} G_{l} c_{0 l}{ }^{\mathrm{T}}+\sum_{r=1}^{n} m_{l r}\left(c_{0 l} G_{l} c_{r l}^{\mathrm{T}}+c_{r l} G_{l} c_{0 l}^{\mathrm{T}}\right),(12) \\
& +\sum_{r, s=1}^{n}\left(m_{l r} m_{l s}+k_{l r s}\right) c_{r l} G_{l} c_{s l}^{\mathrm{T}} \\
H_{l 12}^{(i)}= & c_{0 l} G_{l} d_{0 l}^{(i) \mathrm{T}}+\sum_{r=1}^{n} m_{l r}\left(c_{0 l} G_{l} d_{r l}^{(i) \mathrm{T}}+c_{r l} G_{l} d_{0 l}^{(i) \mathrm{T}}\right),(13) \\
& +\sum_{r, s=1}^{n}\left(m_{l r} m_{l s}+k_{l r s}\right) c_{r l} G_{l} d_{s l}^{(i) \mathrm{T}} \\
H_{l 22}^{(i)}= & d_{0 l}^{(i)} G_{l} d_{0 l}^{(i) \mathrm{T}}+\sum_{r=1}^{n} m_{l r}\left(d_{0 l}^{(i)} G_{l} d_{r l}^{(i) \mathrm{T}}+d_{r l}^{(i)} G_{l} d_{0 l}^{(i) \mathrm{T}}\right) \\
& +\sum_{r, s=1}^{n}\left(m_{l r} m_{l s}+k_{l r s}\right) d_{r l}^{(i)} G_{l} d_{s l}^{(i) \mathrm{T}}
\end{aligned}
$$

$G_{l}$ is the covariance matrix of the random vector $V_{l}$, $m_{l r}(r=1,2, \cdots, n)$, are the components of the vector $m_{l}=E X_{l}, \quad k_{l r s}(r, s=1, \cdots, n)$ are the elements of the covariance matrix $K_{l}=K_{x l}$ of the random vector $X_{l}$. Substitute (10) and (11) into (8) we obtain a sequence of conditionally optimal filtering equations

$$
\hat{X}_{l+1}^{(i)}=a_{l} \hat{X}_{l}^{(i)}+a_{0 l}+\delta_{2 l}^{(i)}\left(Y_{l}^{(i)}-b_{l}^{(i)} \hat{X}_{l}^{(i)}-b_{0 l}^{(i)}\right) .
$$

This is the usual Kalman filtering equation, but in more general problem, Equations (1) and (3) are nonlinear, the matrix coefficient $\delta_{2 l}$ is determined by Formulas (9) and (12)-(14) that are different from the corresponding formula of Kalman linear filtering theory.

Let

$$
\begin{gathered}
m_{l}=E X_{l}, \\
K_{l}=E\left(X_{l}-m_{l}\right)\left(X_{l}-m_{l}\right)^{T}, \\
R_{l}^{(i)}=E \tilde{X}_{l}^{(i)} \tilde{X}_{l}^{(i) T},
\end{gathered}
$$

where $\tilde{X}_{l}^{(i)}=\hat{X}_{l}^{(i)}-X_{l}$ is the error of filtering. To give out the solution of this problem, it is necessary to indicate how the expectation $m_{l}$ and the covariance matrix $K_{l}$ of the random vector $X_{l}$ and the covariance matrix $R_{l}$ of the error of filtering $\tilde{X}_{l}$ can be found at each step. For this purpose, we can deduce from (1) and (15) the stochastic difference equation for the error of filtering $\tilde{X}_{l}$

$$
\begin{aligned}
\tilde{X}_{l+1}^{(i)}= & \left(a_{l}+\delta_{2 l}^{(i)} b_{l}^{(i)}\right) \tilde{X}_{l}^{(i)} \\
& +\left[\delta_{2 l}^{(i)} d_{0 l}^{(i)}-c_{0 l}+\sum_{r=1}^{n}\left(\delta_{2 l}^{(i)} d_{r l}^{(i)}-c_{r l}\right) X_{l r}\right] V_{l}
\end{aligned}
$$

for any $i(i=1, \cdots, N)$. Taking into account that $X_{l}$, 
$\tilde{X}_{l}$ are independent of $V_{l}$ and the unbiaseness of estimate $\hat{X}_{l}^{(i)}\left(E \hat{X}_{l}^{(i)}=E X_{l}\right)$, we can deduce from (1) and (16) that the difference equations for $m_{l}, K_{l}, R_{l}$,

$$
\begin{gathered}
m_{l+1}=a_{l} m_{l}+a_{0 l}, \\
K_{l+1}=a_{l} K_{l} a_{l}^{T}+H_{l 11}, \\
R_{l+1}^{(i)}=a_{l} R_{l}^{(i)} a_{l}^{T}-\delta_{2 l}^{(i)}\left(b_{l}^{(i)} R_{l}^{(i)} a_{l}^{T}+H_{l 12}^{(i) T}\right)+H_{l 11},
\end{gathered}
$$

for any $i(i=1, \cdots, N)$.

Remark $3.1 m_{l}, K_{l}, R_{l}, \delta_{2 l}$ are determined by the Equations (17)-(19) and the Equations (9), (17), (18) are linear difference equations, so $m_{l}, K_{l}$ are determined successively. However, it follows from the formula (9) that $\delta_{2 l}$ depends on $R_{l}$, therefore, Equation (19) is nonlinear difference equation with respect to $R_{l}$.

\subsection{Conditionally Suboptimal Nonlinear Filter}

Next, we start with the suboptimal estimate of conditionally nonlinear filtering $I$, it follows from (15) and (19) that the conditionally nonlinear filtering $I$ has $N$ filtering estimates $\hat{X}_{l}^{(i)}, \hat{X}_{l}^{2}, \cdots, \hat{X}_{l}^{(N)}$ at each step. Then the suboptimal estimate $\hat{X}_{l}^{*}$ for the conditionally nonlinear filtering $I$ is constructed from these estimates $\hat{X}_{l}^{(1)}, \hat{X}_{l}^{(2)}, \cdots, \hat{X}_{l}^{(N)}$ by the following equations

$$
\begin{aligned}
& \hat{X}_{l}^{*}=\sum_{i=1}^{N} p_{l}^{(i)} \hat{X}_{l}^{(i)} \\
& p_{l}^{(i)} \geq 0, \sum_{i=1}^{N} p_{l}^{(i)}=I
\end{aligned},
$$

where $I$ is an unit matrix, $p_{l}^{(1)}, p_{l}^{(2)}, \cdots, p_{l}^{(N)}$ are positive semi-definite matrices and weighted coefficients that are determined by the following mean square criterion,

$$
\min _{\hat{X}_{l}^{*}} E\left\|\hat{X}_{l}^{*}-X_{l}\right\|^{2}=\min _{p_{l}^{(i)}} E\left\|\sum_{i=1}^{N} p_{l}^{(i)} \hat{X}_{l}^{(i)}-X_{l}\right\|^{2} .
$$

Remark 3.2 The suboptimal estimate $\hat{X}_{l}^{*}$ is unbiased. Since each estimate $\hat{X}_{l}^{(i)}(i=1,2, \cdots, N)$ is unbiased, $E X_{l}^{(i)}=E X_{l}(i=1,2, \cdots, N)$, using (20), we can obtain

$$
E \hat{X}_{l}^{*}=\sum_{i=1}^{N} p_{l}^{(i)} E \hat{X}_{l}^{(i)}=\left[\sum_{i=1}^{N} p_{l}^{(i)}\right] E X_{l}=E X_{l} .
$$

\subsection{The Accuracy of Conditionally Suboptimal Nonlinear Filter}

Now, we derive the equation for the actual variance matrix

$$
R_{l}=E\left[\tilde{X}_{l} \tilde{X}^{T}\right], \tilde{X}_{l}=\hat{X}_{l}^{*}-X_{l}
$$

where $X_{l}$ is the state vector (1), $\hat{X}_{l}^{*}$ is the suboptimal filtering estimate (20), and

$$
\tilde{X}_{l}=\sum_{i=1}^{N} p_{l}^{(i)} \tilde{X}_{l}^{(i)}, \tilde{X}_{l}^{(i)}=\hat{X}_{l}^{(i)}-X_{l},
$$

substitute (24) into (23) we can derive that

$$
R_{l}=\sum_{i=1}^{N} p_{l}^{(i)} R_{l}^{(i)} p_{l}^{(i) T}+\sum_{i, j=1, i \neq j}^{N} p_{l}^{(i)} R_{l}^{(i j)} p_{l}^{(j) T},
$$

where $R_{l}^{(i)}$ is determined by Equation (19), $R_{l}^{(i j)}$ is determined by the following formula

$$
\begin{aligned}
R_{l+1}^{(i j)}= & E\left[\tilde{X}_{l}^{(i)} \tilde{X}_{l}^{(j) T}\right] \\
= & \left(a_{l}+\delta_{2 l}^{(i)} b_{l}^{(i)}\right) R_{l}^{(i j)}\left(a_{l}+\delta_{2 l}^{(j)} b_{l}^{(j)}\right)^{T}+Q_{0 l}^{(i)} G_{l} Q_{0 l}^{(j)} \\
& +\sum_{r=1}^{n} m_{l r} Q_{0 l}^{(i)} G_{l} Q_{r l}^{(j) T}+\sum_{r=1}^{n} m_{l r} Q_{r l}^{(i)} G_{l} Q_{0 l}^{(j) T} \\
& +\sum_{r, s=1}^{n} k_{l r s} Q_{r l}^{(i)} G_{l} Q_{s l}^{(j) T} \quad(i \neq j ; i, j=1, \cdots, N),
\end{aligned}
$$

where

$$
Q_{0 l}^{(i)}=\delta_{2 l}^{(i)} d_{0 l}^{(i)}-c_{0 l}, Q_{r l}^{(i)}=\delta_{2 l}^{(i)} d_{r l}^{(i)}-c_{r l}(i=1, \cdots, n)
$$

The question is that there is a sequence of positive semi-definite matrices $p_{l}^{(1)}, p_{l}^{(2)}, \cdots, p_{l}^{(N)}$ such that (21) has a minimal value. Here, we point out that the equations of the optimal coefficients $p_{l}^{(1)}, p_{l}^{(2)}, \cdots, p_{l}^{(N)}$ have the following form,

$$
\begin{aligned}
\frac{\partial}{\partial p_{l}^{(k)}}\left[\operatorname{tr}\left(R_{l}\right)\right]= & \sum_{j=1}^{N} p_{l}^{(j)}\left[R_{l}^{(k j)}-R_{2}^{(N j)}\right]^{T} \\
& +p_{l}^{(N)}\left(R_{l}^{(k N)}-R_{l}^{(N)}\right)^{T} \\
& +\sum_{i=1}^{N-1} p_{2}^{(i)}\left[R_{2 l}^{(i k)}-R_{l}^{(i N)}\right] \\
& +p_{l}^{N}\left(R_{l}^{(N k)}-R_{l}^{(N)}\right)=0,
\end{aligned}
$$

for any $k=1, \cdots, N-1$,

$$
p_{l}^{(1)}+\cdots+p_{l}^{(N)}=I .
$$

The proof of these equations is given in the Appendix. Then the actual variance matrix of the filtering error $R_{l}$ and the actual mean square error $\operatorname{tr}\left(R_{l}\right)$ can be calculated by using the formula (25) and Equations (19), (26), (27). Thus the Equations (9), (12), (13), (14), (19), (26), and (27) completely define the new suboptimal linear filter for estimate $\hat{X}_{l}^{*}$ of the state vector $X_{l}$. Note that the Equations (9), (12), (13), (14), (19), and (26) are separated for any $i(i=1, \cdots, N)$. Therefore, they can be solved in parallel.

\section{Example}

Consider the problem of recursive estimate of an unknown scalar parameter [4]. To estimate the value of the 
unknown parameter $X$ from two types of observations corrupted by additive white noises, the observation models of the stochastic system are given by

$$
\begin{aligned}
& X_{l+1}=a_{l} X_{l}+a_{0 l}+\left(c_{0 l}+c_{1 l} X_{l}\right) V_{l}, \\
& Y_{l}^{(1)}=b_{l}^{(1)} X_{l}+b_{0 l}^{(1)}+\left(d_{0 l}^{(1)}+d_{1 l}^{(1)} X_{l}\right) V_{l}, \\
& Y_{l}^{(2)}=b_{l}^{(2)} X_{l}+b_{0 l}^{(2)}+\left(d_{0 l}^{(2)}+d_{1 l}^{(2)} X_{l}\right) V_{l},
\end{aligned}
$$

where $X_{l}, Y_{l}^{(1)}, Y_{l}^{(2)} \in R$, and $V_{l} \sim N(0,1)$ are independent Gaussian noises, $X_{0} \sim N\left(\hat{X}_{0}, P_{0}\right)$. The optimal filtering estimates $\hat{X}_{l}^{(1)}, \hat{X}_{l}^{(2)}$ based on observation system (30), (31) is determined by the structural functions, respectively,

$$
\begin{aligned}
& \hat{X}_{l+1}^{(1)}=\delta_{1 l}^{(1)} \hat{X}_{l}^{(1)}+\delta_{2 l}^{(1)} Y_{l}^{(1)}+\gamma_{l}^{(1)}, \\
& \hat{X}_{l+1}^{(2)}=\delta_{1 l}^{(2)} \hat{X}_{l}^{(2)}+\delta_{2 l}^{(2)} Y_{l}^{(2)}+\gamma_{l}^{(2)} .
\end{aligned}
$$

Then such this problem is a conditionally nonlinear filtering estimation problem, and applying conditionally nonlinear filtering theory, it follows from (9), (10), (11), (12), (13), and (14) that

$$
\begin{gathered}
m_{l+1}=a_{l} m_{l}+a_{0 l}, \\
K_{l+1}=a_{l} K_{l} a_{l}+H_{l 11},
\end{gathered}
$$

where

$$
H_{l 11}=c_{0 l} G_{l} c_{0 l}+2 m_{l} c_{0 l} G_{l} c_{1 l}+\left(m_{l}^{2}+K_{l}\right) c_{1 l} G_{l} c_{1 l},
$$

and

$$
\begin{gathered}
\delta_{2 l}^{(1)}=\left(a_{l} R_{l}^{(1)} b_{l}^{(1)}+H_{l 12}^{(1)}\right)\left(b_{l}^{(1)} R_{l}^{(1)} b_{l}^{(1)}+H_{l 22}^{(1)}\right)^{-1} \\
R_{l+1}^{(1)}=a_{l} R_{l}^{(1)} a_{l}+H_{l 11}-\delta_{2 l}^{(1)}\left(b_{l}^{(1)} R_{l}^{(1)} a_{l}+H_{l 12}^{(1)}\right), \\
\delta_{1 l}^{(1)}=a_{l}-\delta_{2 l}^{(1)} b_{l}^{(1)}, \\
\gamma_{l}^{(1)}=a_{0 l}-\delta_{2 l}^{(1)} b_{0 l}^{(1)},
\end{gathered}
$$

where

$$
\begin{aligned}
H_{l 12}^{(1)}= & m_{l}\left(c_{0 l} G_{l} d_{1 l}^{(1)}+c_{1 l} G_{l} d_{0 l}^{(1)}\right) \\
& +c_{0 l} G_{l} d_{0 l}^{(1)}+\left(m_{l}^{2}+K_{l}\right) c_{1 l} G_{l} d_{1 l}^{(1)}, \\
H_{l 22}^{(1)}= & m_{l}\left(d_{0 l}^{(1)} G_{l} d_{1 l}^{(1)}+d_{1 l}^{(1)} G_{l} d_{0 l}^{(1)}\right) \\
& +d_{0 l}^{(1)} G_{l} d_{0 l}^{(1)}+\left(m_{l}^{2}+K_{l}\right) d_{1 l}^{(1)} G_{l} d_{1 l}^{(1)},
\end{aligned}
$$

and

$$
\begin{gathered}
\delta_{2 l}^{(2)}=\left(a_{l} R_{l}^{(2)} b_{l}^{(2)}+H_{l 12}^{(2)}\right)\left(b_{l}^{(2)} R_{l}^{(2)} b_{l}^{(2)}+H_{l 22}^{(2)}\right)^{-1}, \\
R_{l+1}^{(2)}=-\delta_{2 l}^{(2)}\left(b_{l}^{(2)} R_{l}^{(2)} a_{l}+H_{l 12}^{(2)}\right)+a_{l} R_{l}^{(2)} a_{l}+H_{l 11}, \\
\delta_{1 l}^{(2)}=a_{l}-\delta_{2 l}^{(2)} b_{l}^{(2)},
\end{gathered}
$$

$$
\gamma^{(2)}=a_{0 l}-\delta_{2 l}^{(2)} b_{0 l}^{(2)},
$$

where

$$
\begin{aligned}
H_{l 12}^{(2)}= & m_{l}\left(c_{0 l} G_{l} d_{1 l}^{(2)}+c_{1 l} G_{l} d_{0 l}^{(2)}\right) \\
& +c_{0 l} G_{l} d_{0 l}^{(2)}+\left(m_{l}^{2}+K_{l}\right) c_{1 l} G_{l} d_{1 l}^{(2)}, \\
H_{l 22}^{(2)}= & m_{l}\left(d_{0 l}^{(2)} G_{l} d_{1 l}^{(2)}+d_{1 l}^{(2)} G_{l} d_{0 l}^{(2)}\right) \\
& +d_{0 l}^{(2)} G_{l} d_{0 l}^{(2)}+\left(m_{l}^{2}+K_{l}\right) d_{1 l}^{(2)} G_{l} d_{1 l}^{(2)} .
\end{aligned}
$$

Next, we will compute conditionally suboptimal nonlinear filtering with two different types of observations and the error variance matrix $R_{l}$. Firstly, we need compute covariance

$$
\begin{aligned}
R_{l+1}^{(12)}= & \left(a_{l}+\delta_{2 l}^{(1)} b_{l}^{(1)}\right) R_{l}^{(12)}\left(a_{l}+\delta_{2 l}^{(2)} b_{l}^{(2)}\right)+Q_{0 l}^{(1)} G_{l} Q_{0 l}^{(2)} \\
& +m_{l} Q_{0 l}^{(1)} G_{l} Q_{0 l}^{(2)}+m_{l} Q_{1 l}^{(1)} G_{l} Q_{0 l}^{(2)}+K_{l} Q_{1 l}^{(1)} G_{l} Q_{1 l}^{(2)}, \\
R_{l+1}^{(21)}= & \left(a_{l}+\delta_{2 l}^{(2)} b_{l}^{(2)}\right) R_{l}^{(21)}\left(a_{l}+\delta_{2 l}^{(1)} b_{l}^{(1)}\right)+Q_{0 l}^{(2)} G_{l} Q_{0 l}^{(1)} \\
& +m_{l} Q_{0 l}^{(2)} G_{l} Q_{0 l}^{(1)}+m_{l} Q_{1 l}^{(2)} G_{l} Q_{0 l}^{(1)}+K_{l} Q_{1 l}^{(2)} G_{l} Q_{1 l}^{(1)},
\end{aligned}
$$

where

$$
\begin{gathered}
Q_{0 l}^{(1)}=\delta_{2 l}^{(1)} d_{0 l}^{(1)}-c_{0 l}, Q_{0 l}^{(2)}=\delta_{2 l}^{(2)} d_{0 l}^{(2)}-c_{0 l}, \\
Q_{1 l}^{(1)}=\delta_{2 l}^{(1)} d_{1 l}^{(1)}-c_{1 l}, Q_{1 l}^{(2)}=\delta_{2 l}^{(2)} d_{1 l}^{(2)}-c_{1 l}
\end{gathered}
$$

Secondly, we will find two weighted coefficients $p_{l}^{(i)}$ $(i=1,2)$ such that the error variance $R_{l}$ is minimal. It follows from Equation (27) at $N=2$ that

$$
\left\{\begin{array}{c}
p_{l}^{(1)}\left[R_{l}^{(1)}-R_{l}^{(12)}\right]+p_{l}^{(2)}\left[R_{l}^{(21)}-R_{l}^{(2)}\right]=0 \\
p_{l}^{(1)}+p_{l}^{(2)}=I,
\end{array}\right.
$$

moreover, we can derive from (51) that

$$
\begin{aligned}
& p_{l}^{(1)}=\frac{R_{l}^{(2)}-R_{l}^{(21)}}{R_{l}^{(1)}+R_{l}^{(2)}-R_{l}^{(12)}-R_{l}^{(21)},} \\
& p_{l}^{(2)}=\frac{R_{l}^{(1)}-R_{l}^{(12)}}{R_{l}^{(1)}+R_{l}^{(2)}-R_{l}^{(12)}-R_{l}^{(21)}},
\end{aligned}
$$

it follows from (20) that the suboptimal estimate

$$
\hat{X}_{l}^{*}=p_{l}^{(1)} \hat{X}_{l}^{(1)}+p_{l}^{(2)} \hat{X}_{l}^{(2)},
$$

and according to (25) the suboptimal estimation variance of error $R_{l}$ has the form:

$$
\begin{aligned}
R_{l}= & p_{l}^{(1)} R_{l}^{(1)} p_{l}^{(1)}+p_{l}^{(2)} R_{l}^{(2)} p_{l}^{(2)} \\
& +p_{l}^{(1)} R_{l}^{(12)} p_{l}^{(2)}+p_{l}^{(2)} R_{l}^{(21)} p_{l}^{(1)},
\end{aligned}
$$

where $R_{l}^{(1)}, R_{l}^{(2)}$ are determined by Equations (38), (44), respectively; and $R_{l}^{(12)}, R_{l}^{(21)}$ are determined by Equations (49), (50), respectively; $p_{l}^{(1)}, p_{l}^{(2)}$ are de- 
termined by the formulas (52), (53), respectively. These equations (38), (44), (49), (50) and the formulas (52), (53), (55) produce the actual accuracy of the conditionally suboptimal nonlinear filtering (29), (30), (31), (54) and (52), (53). Note that $p_{l}^{(1)}, p_{l}^{(2)}$ must be all nonnegative, then

$$
R_{l}^{(1)}-R_{l}^{(12)} \geq 0 \text { and } R_{l}^{(2)}-R_{l}^{(21)} \geq 0 .
$$

However, their expressed forms are quite complicate. Then the simpler example is given as follows: let

$$
\begin{gathered}
b_{l}^{(1)}=0, b_{0 l}^{(1)}=0, b_{l}^{(2)}=0, b_{0 l}^{(2)}=0, \\
d_{l}^{(1)}=0, d_{0 l}^{(2)}=0, a_{0 l}=0, c_{0 l}=0 .
\end{gathered}
$$

Then

$$
\begin{aligned}
& R_{l}^{(1)}=a_{l-1} R_{l-1}^{(1)} a_{l-1}, R_{l}^{(12)}=a_{l-1} R_{l-1}^{(12)} a_{l-1}, \\
& R_{l}^{(2)}=a_{l-1} R_{l-1}^{(2)} a_{l-1}, R_{l}^{(21)}=a_{l-1} R_{l-1}^{(21)} a_{l-1} .
\end{aligned}
$$

When original conditions

$$
R_{0}^{(1)}-R_{0}^{(12)} \geq 0, R_{0}^{(2)}-R_{0}^{(21)} \geq 0,
$$

then

$$
\begin{aligned}
R_{l}^{(1)}-R_{l}^{(12)} & =a_{l-1}\left(R_{l-1}^{(1)}-R_{l-1}^{(12)}\right) a_{l-1} \geq 0, \\
R_{l}^{(2)}-R_{l}^{(21)} & =a_{l-1}\left(R_{l-1}^{(2)}-R_{l-1}^{(21)}\right) a_{l-1} \geq 0 .
\end{aligned}
$$

At this case, substitute all coefficients which are time functions $a_{l}=1 /(l+1)$ and the original conditions $R_{0}^{(1)}=2, \quad R_{0}^{(12)}=1, \quad R_{0}^{(2)}=2.5, \quad R_{0}^{(21)}=1.6 \quad$ into $(57)$ and (58), we can get

$$
R_{l}^{(1)}-R_{l}^{(12)}, R_{l}^{(2)}-R_{l}^{(21)}
$$

furthermore, substitute these into (52) and (53) then we can get $p_{l}^{(1)}, p_{l}^{(2)}$; finally, substitute $p_{l}^{(1)}$ and $p_{l}^{(2)}$ into (55) then we can get $R_{l}$. Numerical simulation results are in Table 1.

Table 1. Numerical values of the optimal estimation variances $R_{l}^{(1)}, R_{l}^{(2)}$ and of the suboptimal estimation variance $R_{l}$ for difference time $l$ when $b_{l}^{(1)}=0, b_{0 l}^{(1)}=0$, $b_{l}^{(2)}=0, \quad b_{0 l}^{(2)}=0, d_{l}^{(1)}=0, d_{0 l}^{(2)}=0, \quad a_{0 l}=0, c_{0 l}=0$, $R_{0}^{(1)}=2, R_{0}^{(12)}=1, R_{0}^{(2)}=2.5, R_{0}^{(21)}=1.6$.

\begin{tabular}{cccc}
\hline$l$ & $R_{l}^{(1)}$ & $R_{l}^{(2)}$ & $R_{l}$ \\
\hline 1 & 2.0 & 2.5 & 1.78947 \\
2 & 0.5 & 0.625 & 0.447368 \\
3 & 0.0555556 & 0.0694444 & 0.0497076 \\
4 & 0.00347222 & 0.00434028 & 0.00310673 \\
5 & 0.000138889 & 0.000173611 & 0.000124269 \\
6 & $3.85802 \times 10^{-6}$ & $4.82253 \times 10^{-6}$ & $3.45192 \times 10^{-6}$ \\
7 & $7.87352 \times 10^{-8}$ & $9.8419 \times 10^{-8}$ & $7.04473 \times 10^{-8}$ \\
8 & $1.23024 \times 10^{-9}$ & $1.5378 \times 10^{-9}$ & $1.10074 \times 10^{-9}$ \\
9 & $1.51881 \times 10^{-11}$ & $1.89851 \times 10^{-11}$ & $1.35894 \times 10^{-11}$ \\
\hline
\end{tabular}

\section{Conclusions}

The new conditionally suboptimal nonlinear filtering is derived for a class of nonlinear discrete systems determined by stochastic difference equations. These stochastic equations all have a parallel structure, therefore, parallel computers can be used in the design of these filters. The numerical example demonstrates the efficiency of the proposed conditionally suboptimal nonlinear filtering. The suboptimal filtering with different types of observations can be widely used in the different areas of applications: military, target tracking, inertial navigation, and others [11].

\section{References}

[1] B. Øksendal, "Stochastic Differential Equations," 6th Edition, Springer-Verlag, Berlin, 2003.

[2] C. Chui and G. Chen, "Kalman Filtering with Real-Time and Applications," Springer-Verlag, Berlin, 2009.

[3] V. I. Shin, "Pugachev's Filters for Complex Information Processing," Automation and Remote Control, Vol. 11, No. 59, 1999, pp. 1661-1669.

[4] F. Lewis, "Optimal Estimation with an Introduction to Stochastic Control Theory," John Wiley and Sons, New York, 1986.

[5] M. Oh, V. I. Shin, Y. Lee and U. J. Choi, "Suboptimal Discrete Filters for Stochastic Systems with Different Types of Observations," Computers \& Mathematics with Applications, Vol. 35, No. 4, 1998, pp. 17-27. doi:10.1016/S0898-1221(97)00275-7

[6] V. S. Pugachev and I. N. Sinitsyn, "Stochastic Systems: Theory and Applications," World Scientific, Singapore, 2002.

[7] I. N. Sinitsyn, "Conditionally Optimal Filtering and Recognition of Signals in Stochastic Differential System," Automation and Remote Control, Vol. 58, No. 2, 1997, pp. 124-130.

[8] Y. Cho, V. I. Shin, M. Oh and Y. Lee, "Suboptimal Continuous Filtering Based on the Decomposition of Observation Vector," Computers \& Mathematics with Applications, Vol. 32, No. 4, 1996, pp. 23-31. doi:10.1016/0898-1221(96)00121-6

[9] Y. Bar-Shalon and L. Campo, "The Effect of the Common Process Noise on the Two-Sensor Fused-Track Covariance," IEEE Transactions on Aerospace and Electronic Systems, Vol. 22, No. 6, 1986, pp. 803-805. doi:10.1109/TAES.1986.310815

[10] I. N. Sinitsyn, N. K. Moshchuk and V. I. Shin, "Conditionally Optimal Filtering of Processes in Stochastic Differential Systems by Bayesian Criteria," Doklady Mathematics, Vol. 47, No. 3, 1993, pp. 528-533.

[11] R. C. Luo and M. G. Kay, "Multisensor Integration and Fusion in Intelligent Systems," IEEE Transactions on Systems, Man and Cybernetics, Vol. 19, No. 5, 1989, pp. 901-931. doi:10.1109/21.44007 


\section{Appendix}

Derivation of Formula (26). It follows from (16) that

$$
\begin{aligned}
R_{l+1}^{(i j)}= & E\left[\tilde{X}_{l+1}^{(i)} \tilde{X}_{l+1}^{(j) T}\right] \\
= & \left(a_{l}+\delta_{2 l}^{(i)} b_{l}^{(i)}\right) E\left[\tilde{X}_{l+1}^{(i)} \tilde{X}_{l+1}^{(j) T}\right]\left(a_{l}+\delta_{2 l}^{(j)} b_{l}^{(j)}\right)^{T} \\
& +\left(\delta_{2 l}^{(i)} d_{0 l}^{(i)}-c_{0 l}\right) E\left(V_{l} V_{l}^{T}\right)\left(\delta_{2 l}^{(j)} d_{0 l}^{(j)}-c_{0 l}\right)^{T} \\
& +\left(\delta_{2 l}^{(i)} d_{0 l}^{(i)}-c_{0 l}\right) E\left[V_{l} V_{l}^{T}\right]\left(\sum_{r=1}^{n}\left(\delta_{2 l}^{(j)} d_{r l}^{(j)}-c_{r l}\right) X_{l r}\right)^{T} \\
& +\left(\sum_{r=1}^{n}\left(\delta_{2 l}^{(i)} d_{r l}^{(i)}-c_{r l}\right) X_{l r}\right) E\left[V_{l} V_{l}^{T}\right] \\
& \left.\cdot\left(\sum_{r=1}^{n} X_{l r}^{T}\left(\delta_{2 l}^{(j)} d_{r l}^{(j)}-c_{r l}\right)\right)^{T}\right) \\
& +\left(\sum_{r=1}^{n}\left(\delta_{2 l}^{(i)} d_{r l}^{(i)}-c_{r l}\right) X_{l r}\right) E\left(V_{l} V_{l}^{T}\right)\left(\delta_{2 l}^{(j)} d_{0 l}^{(j)}-c_{0 l}\right)^{T},
\end{aligned}
$$

set $Q_{0 l}^{(i)}=\delta_{2 l}^{(i)} d_{0 l}^{(i)}-c_{0 l}, \quad Q_{r l}^{(i)}=\delta_{2 l}^{(i)} d_{r l}^{(i)}-c_{r l}$, then it follows from (56) that

$$
\begin{aligned}
R_{l+1}^{(i j)}= & \left(a_{l}+\delta_{2 l}^{(i)} b_{l}^{(i)}\right) R_{l}^{(i j)}\left(a_{l}+a_{l}+\delta_{2 l}^{(i)} b_{l}^{(i)}\right)^{T}+Q_{0 l}^{(i)} G_{l} Q_{0 l}^{(j) T} \\
& +\sum_{r=1}^{n} m_{l r} Q_{0 l}^{(i)} G_{l} Q_{r l}^{(j) T}+\sum_{r=1}^{n} m_{l r} Q_{r l}^{(i)} G_{l} Q_{0 l}^{(j) T} \\
& +\sum_{r=1}^{n} k_{l r s} Q_{r l}^{(i)} G_{l} Q_{s l}^{(j) T} \quad(i \neq j ; i, j=1, \cdots, N) .
\end{aligned}
$$

Derivation of Equation (27). We seek the optimal matrices $p_{l}^{(i)}(i=1, \cdots, N)$ minimizing the mean square error, i.e.,

$$
\begin{gathered}
\operatorname{tr}\left(R_{l}\right)=\sum_{i=1}^{N} \operatorname{tr}\left(p_{l}^{(i)} R_{l}^{(i)} p_{l}^{(i) T}\right)+\sum_{i, j=1, i \neq j}^{N} \operatorname{tr}\left(p_{l}^{(i)} R_{l}^{(i j)} p_{l}^{(j) T}\right) \\
\sum_{i=1}^{N} p_{l}^{(i)}=I, p_{l}^{(i)} \geq 0
\end{gathered}
$$

Next, we use the following formulae

$$
\begin{aligned}
& \frac{\partial}{\partial A} \operatorname{tr}\left(A B A^{T}\right)=A B^{T}+A B, \\
& \frac{\partial}{\partial A} \operatorname{tr}(A B)=\frac{\partial}{\partial A} \operatorname{tr}(B A)=B^{T},
\end{aligned}
$$

to differentiate the function $\operatorname{tr}\left(R_{l}\right)$ with respect to $p_{l}^{(k)}(k=1, \cdots, N-1)$, we can derive that for any $k$,

$$
\begin{aligned}
\frac{\partial}{\partial p_{l}^{(k)}}\left[\operatorname{tr}\left(R_{l}\right)\right]= & \frac{\partial}{\partial p_{l}^{(k)}}\left[\sum_{i=1}^{N} \operatorname{tr}\left(p_{l}^{(i)} R_{l}^{(i)} p_{l}^{(i) T}\right)\right] \\
& +\frac{\partial}{\partial p_{l}^{(k)}}\left[\sum_{i, j=1, i \neq j}^{N} \operatorname{tr}\left(p_{l}^{(i)} R_{l}^{(i j)} p_{l}^{(j) T}\right)\right],
\end{aligned}
$$

substitute (59) into the following equation, we can derive that for any $k$,

$$
\begin{gathered}
\frac{\partial}{\partial p_{l}^{(k)}}\left[\sum_{i=1}^{N} \operatorname{tr}\left(p_{l}^{(i)} R_{l}^{(i)} p_{l}^{(i) T}\right)\right] \\
=2 p_{l}^{(k)} R_{l}^{(k)}-2 R_{l}^{(N)} \\
+2\left(p_{l}^{(1)} p_{l}^{(N)}+\cdots+p_{l}^{(N-1)} p_{l}^{N}\right) \\
=2 p_{l}^{(k)} R_{l}^{(k)}-2 p_{l}^{(N)} R_{l}^{(N)}, \\
\frac{\partial}{\partial p_{l}^{(k)}}\left[\sum_{i, j=1, i \neq j}^{N} \operatorname{tr}\left(p_{l}^{(i)} R_{l}^{(i j)} p_{l}^{(j) T}\right)\right] \\
=\frac{\partial}{\partial p_{l}^{(k)}}\left[\sum_{i, j=1, i \neq j}^{N-1} \operatorname{tr}\left(p_{l}^{(i)} R_{l}^{(i j)} p_{l}^{(j) T}\right)\right] \\
+\frac{\partial}{\partial p_{l}^{(k)}}\left[\sum_{j=1}^{N-1} \operatorname{tr}\left(p_{l}^{(N)} R_{l}^{(N j)} p_{l}^{(j) T}\right)\right] \\
+\frac{\partial}{\partial p_{l}^{(k)}}\left[\sum_{i=1}^{N-1} \operatorname{tr}\left(p_{l}^{(i)} R_{l}^{(i N)} p_{l}^{(N) T}\right)\right] \\
\frac{\partial}{\partial p_{l}^{(k)}}\left[\sum_{i, j=1, i \neq j}^{N-1} \operatorname{tr}\left(p_{l}^{(i)} R_{l}^{(i j)} p_{l}^{(j) T}\right)\right] \\
=\sum_{j=1, j \neq k}^{N-1} p_{l}^{(j)} R_{l}^{(k j) T}+\sum_{i=1, i \neq k}^{N-1} p_{l}^{(i)} R_{l}^{(i k)} .
\end{gathered}
$$

Substitute (59) into the following equations, we can derive that

$$
\begin{aligned}
& \frac{\partial}{\partial p_{l}^{(k)}}\left[\sum_{i=1}^{N-1} \operatorname{tr}\left(p_{l}^{(i)} R_{l}^{(i N)} p_{l}^{(N) T}\right)\right] \\
= & \frac{\partial}{\partial p_{l}^{(k)}}\left[\sum_{i=1}^{N-1} \operatorname{tr}\left(p_{l}^{(i)} R_{l}^{(i N)}\left(I-\sum_{j=1}^{N-1} p_{l}^{(j)}\right)^{T}\right]\right. \\
= & R_{l}^{(k N) T}-p_{l}^{(k)} R_{l}^{(k N) T}-p_{l}^{(k)} R_{l}^{(k N) T} \\
& -\sum_{j=1, j \neq k}^{N-1} p_{l}^{(j)} R_{l}^{(k N) T}-\sum_{i=1, i \neq k}^{N-1} p_{l}^{(i)} R_{l}^{(i N) T}, \\
& \frac{\partial}{\partial p_{l}^{(k)}}\left[\sum_{j=1}^{N-1} \operatorname{tr}\left(p_{l}^{(N)} R_{l}^{(N j)} p_{l}^{(j) T}\right)\right] \\
= & \frac{\partial}{\partial p_{l}^{(k)}}\left[\sum_{i=1}^{N-1} \operatorname{tr}\left(\left(I-\sum_{j=1}^{N-1} p_{l}^{(j)}\right) R_{l}^{(N j)} p_{l}^{(j) T}\right]\right. \\
= & R_{l}^{(N k)}-p_{l}^{(k)}\left[R_{l}^{(k N)}\right]^{T}-p_{l}^{(k)} R_{l}^{(N k)} \\
& -\sum_{j=1, j \neq k}^{N-1} p_{l}^{(j)}\left[R_{l}^{(N j)}\right]^{T}-\sum_{i=1, i \neq k}^{N-1} p_{l}^{(i)} R_{l}^{(N k)}
\end{aligned}
$$

for any $k \quad(k=1, \cdots, N-1)$. Substitute (62)-(66) into (61), we can derive that 


$$
\begin{aligned}
\frac{\partial}{\partial p_{l}^{(k)}}\left[\operatorname{tr}\left(R_{l}\right)\right] & =\sum_{j=1, j \neq k}^{N-1} p_{l}^{(j)}\left[R_{l}^{(k j)}-R_{l}^{(N j)}\right]^{T}+p_{l}^{(k)}\left[R_{l}^{(k k)}-R_{l}^{(N k)}\right]^{T} \\
& +\sum_{i=1, i \neq k}^{N-1} p_{l}^{(i)}\left[R_{l}^{(i k)}-R_{l}^{(i N)}\right]+p_{l}^{(k)}\left[R_{l}^{(k k)}-R_{l}^{(k N)}\right] \\
& -\sum_{j=1}^{N-1} p_{l}^{(j)}\left(R_{l}^{(k N)}\right)^{T}-\sum_{i=1}^{N-1} p_{l}^{(i)}\left(R_{l}^{(k N)}\right)^{T}+\left(R_{l}^{(k N)}\right)^{T}+R_{l}^{(N k)}-2 p_{l}^{(N)} R_{l}^{(N N)} \\
= & \sum_{j=1}^{N-1} p_{l}^{(j)}\left[R_{l}^{(k j)}-R_{l}^{(N j)}\right]^{T}+p_{l}^{(N)}\left(R_{l}^{(k N)}-R_{l}^{(N)}\right)^{T} \\
& +\sum_{i=1}^{N-1} p_{l}^{(i)}\left[R_{l}^{(i k)}-R_{l}^{(i N)}\right]+p_{l}^{(N)}\left(R_{l}^{(N k)}-R_{l}^{(N)}\right)
\end{aligned}
$$

let the result be zero, we get (27). 